Journal of Nonlinear Optical Physics \& Materials

Vol. 14, No. 4 (2005) 581-588

(C) World Scientific Publishing Company

\title{
AUTHOR INDEX VOLUME 14
}

Ahn J-H, see Kang $Y$

14 (2005) 487

Akhmediev N, Soto-Crespo JM, Grapinet M \& Grelu Ph, Dissipative Soliton Pulsations with Periods Beyond the Laser Cavity Round Trip Time

$14(2005) 177$

Alatas H, Iskandar AA, Tjia MO \& Valkering TP, Optical Sensing and Switching Device Based on a Finite Deep Nonlinear Bragg Grating with a Mirror

Amari N, see Barsu $C$

Andraud C, see Barsu $C$

Andraud C, see Lin C-L

Antipin M, see Sarkisov SS

Apanasevich PA, see Lisinetskii VA

Bae B-S, see Yoon KB

Bae $\mathrm{SH}$, see Hwang EJ

Baek J-J, see Kim DU

Baek JJ, Jeong YC, Park LS, Lee JK, Han YS \& Kwon Y, Synthesis and Electro-Optical Properties of Blue Emitting Poly(BP-alt-BCV) Conjugated Polymers

Baek JJ, see Jeong JW

Baek NS, see Kim HK

Baldeck PL, see Barsu $C$

Baldeck PL, see Lin $C-L$

Bananej A \& Li C, Parameter Controllable All-Optical Switching in a High-Nonlinear Micro Ring Coupled MZI Through a Pumped Nonlinear Coupler

Barsu C, Andraud C, Amari N, Spagnoli S \& Baldeck PL, Dendritic Fluorene Oligomers for Nonlinear Absorption in the Visible Range

Beaudin AMR, see Song $N$

Bigot J-Y, see Lincker $F$

Boiko Y, Z-Scan Approach for Measuring the Threshold of Two-Photon Photopolymerization

Boudebs G, see Cherukulappurath $S$

Boyd RW, see Piredda $G$

Carter RR, see Kost AR

Chen M, Li C, Ma S, Xu M, Wang W \& Xia Y, Optical Bistability Switching Property in One-Dimensional Nonlinear Photonic Crystal

14 (2005) 259

14 (2005) 311

14 (2005) 311

14 (2005) 375

14 (2005) 21

14 (2005) 107

14 (2005) 399

14 (2005) 475

14 (2005) 529

14 (2005) 535

14 (2005) 545

14 (2005) 555

14 (2005) 311

14 (2005) 375

$14(2005) 85$

14 (2005) 311

14 (2005) 367

14 (2005) 319

14 (2005) 79

14 (2005) 49

14 (2005) 9

14 (2005) 449

$14(2005) 41$ 
Chen M, Li C, Xu M, Wang W, Ma S, Xia Y \& Liu D, Dynamical Addressing Optical Interconnection Based on One-Dimensional Nonlinear Photonic Crystal

$14(2005) 461$

Cherukulappurath S, Godet JL \& Boudebs G, Higher Order Coefficient Measurements in Nonlinear Absorption Process

Chiang KS, Theory of Pulse Propagation in Optical Directional Couplers

Chin M-K, see Mario $L Y$

Chin MK, see Darmawan $S$

Cho HN, see Lee SE

Cho K-H, Rhee BK, Sasaki Y \& Ito H, Pulsed Intracavity Optical Parametric Oscillator with High Average Power Based on Periodically Poled $\mathrm{LiNbO}_{3}$

Cho KH, see Lee GJ

Choi C-G, see Kee $C$-S

Choi DH, see Jun $W G$

Choi HS, Park JW, Park LS, Lee JK, Han YS \& Kwon Y, Preparation of Panel and Charged Particles for Electrophoretic Display

Chulkov RV, see Lisinetskii VA

Chung G, see Shin D-M

Colombier I, see Lin $C$ - $L$

Curley MJ, see Sarkisov SS

Darmawan S, Lee SY, Lee CW \& Chin MK, Transformation of Directional Couplers to Multi-Mode Interferometers Based on Ridge Waveguides and Its Applications

Didier P, see Lincker F

Dorrer C, see Piredda $G$

Driessen A, see Tan FS

Ehsan AA, see Razali $N$

Eichler H-J, see Lisinetskii VA

Elim HI, see Yuwono $A H$

Fetterman HR, see Kim $S$

Fleitz PA, see Sarkisov SS

Folliot $\mathrm{H}$, see Lecourt $J-B$

Gal Y-S, see Lee $S-G$

Gao JP, see Song $N$

Gao Y, see Hsu $H$

Garmire EM, see Kost AR

Geary K, see Kim $S$

Glavcheva Z, Umezawa H, Okada S \& Nakanishi H, New Trimethylammonium Derivatives Toward Second-Order Nonlinear Optics

Godet JL, see Cherukulappurath $S$

Grabtchikov AS, see Lisinetskii VA

Grapinet M, see Akhmediev $N$

Grelu $\mathrm{Ph}$, see Akhmediev $N$

Grishina AD, see Vannikov $A V$

van Groesen E, see Nicolau $J B$

van Groesen E, see Suryanto A

Gu B-Y, see Zhao $L-M$

14 (2005) 49

$14(2005) 133$

$14(2005) 245$

$14(2005) 221$

$14(2005) 469$

14 (2005) 383

14 (2005) 305

14 (2005) 299

14 (2005) 497

$14(2005) 521$

14 (2005) 107

14 (2005) 513

14 (2005) 375

14 (2005) 21

14 (2005) 221

14 (2005) 319

14 (2005) 9

14 (2005) 273

14 (2005) 195

14 (2005) 107

14 (2005) 281

14 (2005) 391

14 (2005) 21

14 (2005) 427

14 (2005) 573

14 (2005) 367

14 (2005) 1

14 (2005) 449

14 (2005) 391

14 (2005) 357

14 (2005) 49

14 (2005) 107

14 (2005) 177

14 (2005) 177

14 (2005) 439

14 (2005) 161

14 (2005) 203

14 (2005) 115 
Guidoni L, see Lincker $F$

14 (2005) 319

Guézo $\mathrm{M}$, see Lecourt $J$ - $B$

14 (2005) 427

Hammer M, see Suryanto $A$

14 (2005) 203

Han SP, see Kee C-S

14 (2005) 299

Han YS, see Baek JJ

14 (2005) 535

Han YS, see Choi HS

14 (2005) 521

Han YS, see Jeong JW

14 (2005) 545

Han YS, see Kim DU

$14(2005) 529$

Hasenberg TC, see Kost AR

14 (2005) 449

Heo J, see Kang $Y$

Hideur A, see Lecourt $J$-B

Ho TK, see Lue $Y Q$

Hong J-S, see Park JK

Hong K-S, see Kim HK

Hsu H, Li TN, Yang EZ, Yu JL, Lü J, Xu Y \& Gao Y, Effect of

Phonons in Stimulated Brillouin Scattering on Optical Fiber

Communication

Hwang EJ, Bae SH, Lee JH, Kim TW \& Park JW, Synthesis and

Characterization of Long-Alkyl Chained Carbazole Derivatives

Iskandar AA, see Alatas $H$

Ito $\mathrm{H}$, see $\mathrm{Cho} K-\mathrm{H}$

Jeong JW, Kwon Y, Baek JJ, Park LS, Lee E-W, Han YS \& Kim HT, Synthesis and Electroluminescent Properties of

Polyazomethine-Type Conjugated Polymers Containing Heterocyclic Phenothiazine and Carbazole Moiety

Jeong YC, see Baek JJ

Jeong YC, see Kim DU

Jeong YH, see Lee GJ

$\mathrm{Ji} \mathrm{W}$, see Yuwono $A H$

Jin S-H, see Lee $S-G$

Ju H-J, see Kim DU

Jun WG, Yoon H, Lee SH, Kim JH \& Choi DH, Inorganic-Organic

14 (2005) 487

14 (2005) 427

14 (2005) 93

14 (2005) 565

14 (2005) 555

$14(2005) 1$

14 (2005) 475

14 (2005) 259

14 (2005) 383

Hybrid Photorefractive Materials bearing the Bifunctional

Chromophore

Jung SH, see Lee $S E$

Jung SO, see Kang $Y$

Jung W-J, see Kim $S$

Jung W-T, see Lee $J$ - $Y$

Ka J-W, see Kim HK

Kang S-J, see Kim S

Kang Y, Park J, Heo J, Park K-M, Ahn J-H, Jung SO, Kim Y-H \&

Kwon S-K, Toward Efficient Electron-Transporting and

Blue-Emitting Materials for Organic Light-Emitting Diodes:

Structure and Photoluminescent Properties of Silole

Derivatives

Kee C-S, Yoon KB, Choi C-G, Kim J-T, Han SP, Park S \& Schift H, Nanopatterned Polymer Thin Films

Kelderman H, see Tan FS

Kim A, Nonlinear Optical Processes Producing Three-Fold

Entanglements Via $\chi^{(2)}$ and $\chi^{(3)}$ Material

$14(2005) 545$

$14(2005) 535$

$14(2005) 529$

$14(2005) 305$

$14(2005) 281$

14 (2005) 573

14 (2005) 529

14 (2005) 497

14 (2005) 469

14 (2005) 487

14 (2005) 391

14 (2005) 341

14 (2005) 555

14 (2005) 391

14 (2005) 487

14 (2005) 299

14 (2005) 273

14 (2005) 67 
Kim DU, Paik S-H, Kim S-H, Tak Y-H, Kim S-D, Kim K-D, Ju H-J, Kim T-J, Baek J-J, Jeong YC, Park LS \& Han YS, Iridium Complexes with 3-Methyl-2,4-Pentanedione Ligand for Organic Electroluminescent Device

$14(2005) 529$

$14(2005) 305$

Kim EK, see Lee GJ

Kim HK, Baek NS, Oh JB, Ka J-W, Roh S-G, Kim YH, Nah MK, Hong K-S, Song BJ \& Zhou G, Lanthanide(III)-Cored

Supramolecular Complexes with Light-Harvesting Dendritic Arrays for Advanced Photonics Applications

Kim HK, see Park JK

Kim HT, see Jeong JW

Kim J-T, see Kee $C$-S

Kim JH, see Jun $W G$

Kim JK, see Park JH

Kim JY, see Park JH

Kim K, see Shin D-M

Kim K-D, see Kim DU

Kim S, Geary K, Yuan W, Fetterman HR, Zhang C, Wang C, Steier WH, Park G-C, Kang S-J, Oh I \& Jung W-J, Metal-Defined Passive Polymer Optical Waveguides Operating at Both 1.31 and $1.55 \mu \mathrm{m}$ Wavelengths

Kim S-D, see Kim DU

Kim S-H, see Kim DU

Kim T-J, see Kim DU

Kim TW, see Hwang EJ

Kim WS, Lim JH, Lee B-J, Oh Y \& Kwon Y-S, Synthesis and Electroluminescent Property of Aluminum and $\alpha$-Pyridoin Complex Kim Y-H, see Kang $Y$

Kim YC, see Park JH

Kim YH, see Kim HK

Knoll W, see $Y u F$

Kong HJ, see Park SH

Kost AR, Carter RR, Garmire EM \& Hasenberg TC, Nonlinear Optical Properties of a Hetero-nipi Structure with Coupled Quantum Wells

Krivenko TV, see Vannikov AV

Kuang CS, see Razali $N$

Kwak Y-W, see Lee $S$ - $G$

Kwon S-K, see Kang $Y$

Kwon Y, see Baek JJ

Kwon Y, see Choi HS

Kwon Y, see Jeong JW

Kwon Y-S, see Kim WS

Labbé C, see Lecourt $J$-B

Lecourt J-B, Ortaç B, Guézo M, Labbé C, Folliot H, Loualiche S, Hideur A \& Martel G, Highly Fe-Doped InGaAs/InP Saturable Absorber Mode-Locking of an Erbium Fiber Laser

Lee B-J, see Kim WS

Lee CJ, see Lee $S E$

Lee CW, see Darmawan $S$
$14(2005) 555$

$14(2005) 565$

$14(2005) 545$

14 (2005) 497

14 (2005) 481

14 (2005) 481

14 (2005) 513

14 (2005) 529

14 (2005) 391

14 (2005) 529

14 (2005) 529

14 (2005) 529

14 (2005) 475

14 (2005) 505

14 (2005) 487

14 (2005) 481

14 (2005) 555

14 (2005) 149

14 (2005) 331

$14(2005) 449$

14 (2005) 439

14 (2005) 195

14 (2005) 573

14 (2005) 487

14 (2005) 535

14 (2005) 521

14 (2005) 505

14 (2005) 427

14 (2005) 427

14 (2005) 505

14 (2005) 469

$14(2005) 221$
14 (2005) 299

14 (2005) 545 
Lee D-H, see Park JK

$14(2005) 565$

Lee E-H, Lee S-G, O BH \& Park S-G, Polymer-Based Optical Printed Circuit Board $(O-P C B)$ as a Potential Platform for VLSI Microphotonic Integration

$14(2005) 409$

Lee E-W, see Jeong JW

Lee GJ, Jeong YH, Lee JJ, Oh CH, Kim EK, Lee YP, Cho KH \& Shin DW, Photonic Pattern Fabrication in Fused Silica, BK7, and Ge-Doped Borophosphosilica Glass by a Femtosecond Laser

Lee J-Y, Jung W-T \& Rhee BK, Synthesis of Novel Y-Type

Polyurethanes with High Thermal Stability of Dipole Alignment and Their Electro-Optic Properties

Lee $\mathrm{JH}$, see Hwang EJ

Lee JJ, see Lee GJ

Lee JK, see Baek JJ

Lee JK, see Choi HS

Lee K-S, see Park $S H$

Lee S-G, Kwak Y-W, Park J-W, Jin S-H \& Gal Y-S, Synthesis of Poly(Dipropargylfluorene) by the Palladium-Catalyzed Cross Coupling Reaction and its Luminescent Properties

Lee S-G, see Lee $E$ - $H$

Lee SE, Cho HN, Jung SH, Park HC, Lee CJ \& Park JW, Novel Synthesis of Highly Phenyl-Substituted Spirobifluorene and Carbazole Derivatives through Diels-Alder Reaction for Light-Emitting Diodes

Lee $\mathrm{SH}$, see Jun $W G$

Lee SY, see Darmawan $S$

Lee YP, see Lee GJ

Leyderman A, see Sarkisov SS

Li C, see Bananej $A$

Li C, see Chen $M$

Li C, see Chen $M$

Li TN, see Hsu $H$

Lim JH, see Kim WS

Lim TW, see Park $S H$

Lin C-L, Wang I, Pierre M, Colombier I, Andraud C \& Baldeck PL, Rotational Properties of Micro-Slabs Driven by Linearly-Polarized Light

14 (2005) 545

14 (2005) 305

14 (2005) 341

14 (2005) 475

14 (2005) 305

14 (2005) 535

14 (2005) 521

14 (2005) 331

14 (2005) 573

14 (2005) 409

14 (2005) 469

14 (2005) 497

14 (2005) 221

14 (2005) 305

14 (2005) 21

14 (2005) 85

14 (2005) 41

14 (2005) 461

14 (2005) 1

14 (2005) 505

14 (2005) 331

$14(2005) 375$

Lincker F, Masson P, Nicoud J-F, Didier P, Guidoni L \& Bigot J-Y, Synthesis and Characterization of Efficient Two-Photon Absorption Chromophores with Increased Dimensionality

Lisinetskii VA, Mishkel' II, Chulkov RV, Grabtchikov AS, Apanasevich PA, Eichler H-J \& Orlovich VA, Raman Gain Coefficient of Barium Nitrate Measured for the Spectral Region of Ti:Sapphire Laser

Liu D, see Chen $M$

Loualiche S, see Lecourt J-B

Lü J, see Hsu $H$

Lue JT, see Lue $Y Q$

Lue YQ, Ho TK, Pang CP \& Lue JT, Study on the Optical Second-Order Nonlinearity of Submicrospherical Silica Glass

$14(2005) 107$

14 (2005) 461

14 (2005) 427

14 (2005) 1

14 (2005) 93

14 (2005) 93 
Ma S, see Chen $M$

$14(2005) 41$

Ma S, see Chen $M$

$14(2005) 461$

Mario LY, Yuan X \& Chin M-K, Reversal of Interference in Left Handed Medium

$14(2005) 245$

Martel G, see Lecourt J-B

$14(2005) 427$

Masson P, see Lincker $F$

Men L, see Song $N$

Mishkel' II, see Lisinetskii VA

Mohamed R, see Razali $N$

Nah MK, see Kim HK

Nakanishi H, see Glavcheva Z

Nesterov VN, see Sarkisov SS

Ngurah Made DP, Sahar MR \& Sudin Md R, Interrelation of

Intrinsic Defects and Optical Absorption Properties of Lithium

Niobate $\left(\mathrm{LiNbO}_{3}\right)$ Crystals

Nicolau JB \& van Groesen E, Hybrid Analytic-Numeric Method for

Light through a Bounded Planar Dielectric Structure

Nicoud J-F, see Lincker $F$

$\mathrm{O} \mathrm{BH}$, see Lee $\mathrm{E}-\mathrm{H}$

$\mathrm{Oh} \mathrm{CH}$, see Lee GJ

Oh I, see Kim $S$

Oh JB, see Kim HK

Oh JB, see Park JK

Oh Y, see Kim WS

Okada S, see Glavcheva $Z$

Orlovich VA, see Lisinetskii VA

Ortaç B, see Lecourt J-B

Paik S-H, see Kim DU

Pang CP, see Lue $Y Q$

Park G-C, see Kim $S$

Park HC, see Lee SE

Park J, see Kang $Y$

Park J-W, see Lee $S$ - $G$

Park JH, Park OO, Kim JK, Yu J-W, Kim JY \& Kim YC,

White-Electroluminescence Device Based on Polymer/Quantum Dot

Nanocomposites

$14(2005) 319$

14 (2005) 367

14 (2005) 107

14 (2005) 195

14 (2005) 555

14 (2005) 357

14 (2005) 21

14 (2005) 237

14 (2005) 161

14 (2005) 319

14 (2005) 409

14 (2005) 305

14 (2005) 391

14 (2005) 555

14 (2005) 565

14 (2005) 505

14 (2005) 357

14 (2005) 107

14 (2005) 427

14 (2005) 529

14 (2005) 93

14 (2005) 391

14 (2005) 469

14 (2005) 487

14 (2005) 573

14 (2005) 481

Park JK, Song BJ, Lee D-H, Song Y-B, Oh JB, Hong J-S \& Kim HK,

Thermally Stable Perfluorinated Polyimides with Pendant

Perfluorinated Aromatic Unit for Photonic Applications

$14(2005) 565$

Park JW, see Choi HS

Park JW, see Hwang EJ

$14(2005) 521$

$14(2005) 475$

$14(2005) 469$

Park JW, see Lee $S E$

$14(2005) 487$

$14(2005) 535$

$14(2005) 521$

14 (2005) 545

$14(2005) 529$

$14(2005) 481$

Park OO, see Park JH

$14(2005) 299$

Park S, see Kee C-S

$14(2005) 409$

Park S-G, see Lee E-H 
Park SH, Lim TW, Yang D-Y, Yi SW, Kong HJ \& Lee K-S, Direct Nano-Patterning Methods Using Nonlinear Absorption in

Photopolymerization Induced by a Femtosecond Laser

$14(2005) 331$

Pereshivko L Ya, see Vannikov AV

Peterson BH, see Sarkisov SS

Pierre $\mathrm{M}$, see Lin $C$ - $L$

Piredda G, Dorrer C, Williams EMK, Walmsley IA \& Boyd RW, Measurement of the Intensity-Dependent Refractive Index Using Complete Spatio-Temporal Pulse Characterization

$14(2005) 439$

$14(2005) 21$

$14(2005) 375$

$14(2005) 9$

$14(2005) 399$

$14(2005) 21$

Radovanova EI, see Sarkisov SS

Razali N, Mohamed R, Ehsan AA, Kuang CS \& Shaari S, Thermo-Optic Coefficient of Different Photosensitive Acrylate Polymers for Optical Application

Rhee BK, see Cho $K-H$

Rhee BK, see Lee $J-Y$

Roh S-G, see Kim HK

Rychwalski RW, see Vannikov $A V$

Sahar MR, see Ngurah Made DP

Sarkisov SS, Peterson BH, Curley MJ, Nesterov VN, Timofeeva T, Antipin M, Radovanova EI, Leyderman A \& Fleitz PA, Two-Photon Absorption and Fluorescence of New Derivatives of Cyclohexanone and Piperidone

Sasaki Y, see Cho K-H

Savelyev VV, see Vannikov AV

Schift H, see Kee $C$-S

Shaari S, see Razali $N$

Shin D-M, Song D-M, Chung G \& Kim K, Complexation of Aromatic Molecules with New Calixarene Derivatives Containing Two Aryl Sulfide Rings

Shin DW, see Lee GJ

Song BJ, see Kim HK

Song BJ, see Park JK

Song D-M, see Shin D-M

Song N, Men L, Gao JP, Yu G, Beaudin AMR \& Wang ZY, Towards Thermally Stable, Highly Electro-Optically Active Organic Polymers: Design and Synthesis of Crosslinkable Polyimides Containing Zwitterionic Nonlinear Optical Chromophores

Song Y-B, see Park JK

Soto-Crespo JM, see Akhmediev $N$

Spagnoli S, see Barsu $C$

Steier WH, see Kim $S$

Sudin Md R, see Ngurah Made DP

Suryanto A, van Groesen E \& Hammer M, Weakly Nonparaxial Effects on the Propagation of $(1+1) D$ Spatial Solitons in Inhomogeneous Kerr Media

Tak Y-H, see Kim DU

Tan FS, Kelderman H \& Driessen A, Ultra-Compact Spectral Slicer Devices Based on Microring Resonators

Timofeeva T, see Sarkisov SS

$14(2005) 195$

14 (2005) 383

14 (2005) 341

14 (2005) 555

14 (2005) 439

14 (2005) 237

14 (2005) 21

14 (2005) 383

14 (2005) 439

14 (2005) 299

14 (2005) 195

14 (2005) 513

14 (2005) 305

14 (2005) 555

14 (2005) 565

14 (2005) 513

14 (2005) 367

14 (2005) 565

$14(2005) 177$

14 (2005) 311

14 (2005) 391

14 (2005) 237

14 (2005) 203

14 (2005) 529

14 (2005) 273

14 (2005) 21 
Tjia MO, see Alatas $H$

Umezawa H, see Glavcheva Z

$14(2005) 259$

Valkering TP, see Alatas $H$

$14(2005) 357$

$14(2005) 259$

Vannikov AV, Grishina AD, Pereshivko L Ya, Krivenko TV, Savelyev VV \& Rychwalski RW, Infrared Photorefractive Composites Based on Polyimide and J-Aggregates of Cyanine Dye

Walmsley IA, see Piredda $G$

Wang C, see Kim $S$

Wang I, see Lin $C-L$

Wang J, see Yuwono AH

Wang W, see Chen $M$

Wang W, see Chen $M$

Wang ZY, see Song $N$

Williams EMK, see Piredda $G$

Wu W, Zhang Z \& Zhang X, Design and Syntheses of Highly Efficient and Thermally-Stable Imidazole Chromophores Bearing Diamino Groups for Nonlinear Optics

Xia Y, see Chen $M$

Xia Y, see Chen $M$

$\mathrm{Xu} \mathrm{M}$, see Chen $M$

$\mathrm{Xu} \mathrm{M}$, see Chen $M$

$\mathrm{Xu} \mathrm{Y}$, see $H$ su $H$

Xue J, see Yuwono AH

Yang D-Y, see Park $S H$

Yang EZ, see Hsu $H$

Yang G-Z, see Zhao $L-M$

Yi SW, see Park $S H$

Yoon $\mathrm{H}$, see Jun $W G$

Yoon KB, Bae B-S \& Popall M, Fabrication of Low-Loss Waveguides Using Organic-Inorganic Hybrid Materials

Yoon $\mathrm{KB}$, see Kee $C$-S

Yu F \& Knoll W, Surface Plasmon Diffraction Biosensor

$\mathrm{Yu}$ G, see Song $N$

Yu J-W, see Park JH

Yu JL, see Hsu $H$

Yuan W, see Kim $S$

Yuan X, see Mario LY

Yuwono AH, Xue J, Wang J, Elim HI \& Ji W, Transparent $\mathrm{TiO}_{2}-\mathrm{PMMA}$ Nanohybrids of High Nanocrystallinity and Enhanced Nonlinear Optical Properties

Zhang C, see Kim $S$

Zhang X, see $W u W$

Zhang Z, see $W u W$

Zhao L-M, Gu B-Y, Yang G-Z \& Zhou Y-S, Optimal Design of Aperiodic Optical Superlattices for Achieving Parametric Amplification or Second Harmonic Generation with Consideration of the Depletion of Pumping Light Power

Zhou G, see Kim HK

Zhou Y-S, see Zhao L-M

$14(2005) 439$

14 (2005) 9

14 (2005) 391

14 (2005) 375

14 (2005) 281

14 (2005) 41

14 (2005) 461

14 (2005) 367

14 (2005) 9

14 (2005) 61

14 (2005) 41

14 (2005) 461

14 (2005) 41

14 (2005) 461

14 (2005) 1

14 (2005) 281

14 (2005) 331

14 (2005) 1

14 (2005) 115

14 (2005) 331

14 (2005) 497

14 (2005) 399

14 (2005) 299

14 (2005) 149

14 (2005) 367

14 (2005) 481

14 (2005) 1

14 (2005) 391

14 (2005) 245

14 (2005) 281

14 (2005) 391

14 (2005) 61

14 (2005) 61

14 (2005) 115

14 (2005) 555

14 (2005) 115 